\title{
MEASUREMENT OF YOUNG'S MODULUS ON MICROFABRICATED STRUCTURES USING A SURFACE PROFILER
}

\author{
Yu-Chong Tai** and Richard S. Muller \\ Berkeley Sensor and Actuator Center \\ An NSF/Industry/University Cooperative Research Center \\ Department of EECS and the Electronics Research Laboratory \\ University of California, Berkeley CA 94720 \\ ** Present Address: Mail Stop 116-81 \\ California Institute of Technology, Pasadena, CA 91125
}

\begin{abstract}
A point-force, load-deflection method using a stylus-type surface profiler to determine the Young's moduli $\left(E_{Y}\right)$ of thinfilm microstructural materials is introduced. In this method both force and deflection in microstructures are measured simultaneously by the profiler to provide a convenient and accurate means to obtain Young's modulus directly. Measurements on two types of micromechanical structures are described: a doubly supported bridge and a bridge-slider (a beam with one fixed end and one end which can slide in a flanged housing). The influence of residual stress in the doubly supported beam is described and accounted for theoretically to interpret measurements made on low-stress silicon-nitride films. For this material $E_{Y}$ is $373 \mathrm{GPa}$. In the bridge-slider structure, residual strain is relaxed to zero. Measurements on a polycrystalline-silicon bridge slider show a value for $E_{Y}$ of $123 \mathrm{GPa}$ in unannealed material that is doped heavily with phosphorus, and grown at $650^{\circ} \mathrm{C}$.
\end{abstract}

\section{INTRODUCTION}

Three methods have been identified in previous research as useful to measure the Young's moduli of thin-film materials. The first of these makes use of measured wafer warpage under the stress imposed by coating with the thin-film material. Typically, either a range of coating thicknesses are used [1], or else the temperature is varied [2]. A second method uses

measurements made on the deflection of membranes of the thin-film material that are subjected to differential pressures $[3,4]$. The third technique is based upon measurements of the resonant frequency $f_{r}$ of vibrating structures such as cantilevers or doubly supported bridges [5-7]. Young's modulus is extracted from the theoretical expression for $f_{r}$. All three of these methods are relatively complicated and painstaking to carry out.

We report here a straightforward and relatively easy point-force, load-deflection method to obtain $E_{Y}$ which requires only a stylus-type surface profiler, a test machine that is present in most microfabrication laboratories. In this new method, the stylus of the surface profiler scans over a free-standing structure made of the material under test. The profiler records both the stylus force and the vertical position of the free-standing struc- ture under load. A mechanical analysis is used to determine the dependence of the structural deflection in terms of $E_{Y}$ for the material including the effects of residual strain, if any. The measured data is then used to extract a value for the Young's modulus of the material.

\section{THEORY}

Figure 1 shows the cross section of an arbitrarily shaped free-standing structure (an example might be a microbridge) which is loaded by a stylus force $F$. In the linear bending region (small deflection), the height $h$ of the free-standing structure can be related to the loading force $F$ by

$$
h_{o}-h=\frac{F}{E_{Y}} K
$$

where $h_{o}$ and $h$ are the heights of the unloaded and loaded structure respectively, $E_{Y}$ is the Young's modulus of the structural material, and $K$ is a proportionality constant with units of reciprocal length. The constant $K$ is a function of the geometric sizes, boundary conditions, Poisson's ratio, and the residual stress of the structure. From Eq. (1), we can readily derive

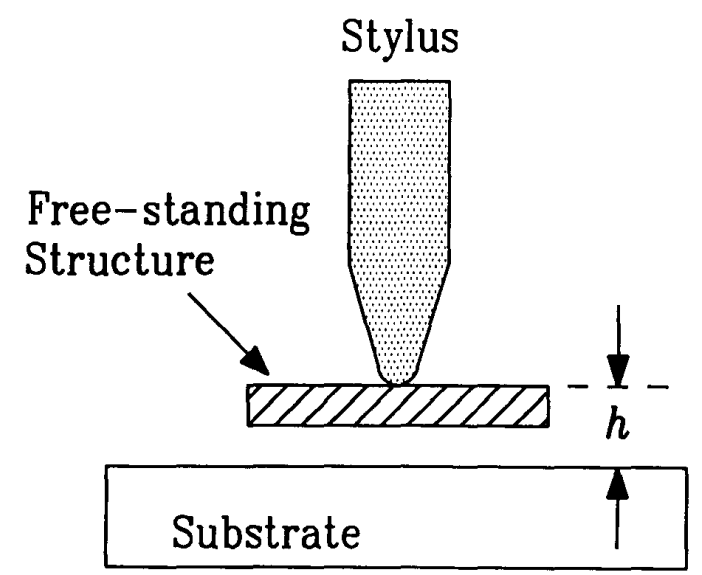

Figure 1 Elements of the point-force, load-deflection method for Young's modulus measurement. 


$$
E_{Y}=-\frac{K}{\frac{d h}{d F}}
$$

In Eq. (2), two terms, $\frac{d h}{d F}$ and $K$, are needed to calculate $E_{Y}$. We shall show that the slope $\frac{d h}{d F}$ is easily measured using a surface profiler. We have calculated the proportionality constant $K$ for the microstructures tested using a two-dimensional, finite-element simulator, SUPERSAP [8].

\section{EXPERIMENTAL PROCEDURE}

The surface profiler used in our experiments is an AlphaStep 200 [9]. This profiler has a stylus force that can be adjusted from 1-25 mg, but the precision of this loading is poorer than $\pm 20 \%$, especially in the lower range of loads. The force range is suitable for loading of microstructures, but the load inaccuracy is unacceptable for $E_{Y}$ determination. We have therefore used micromechanics to construct a gauge which measures the stylus force to an accuracy of $\pm 0.1 \mathrm{mg}$ [10].

Table 1 shows the actual stylus force measured by the microgauge as a function of different settings on the AlphaStep 200 profiler.

Table 1 Calibrated readout of the stylus forces.

\begin{tabular}{|c|c|l|}
\hline Force Setting (mg) & Measured & Offset \\
\hline 1 & 1.2 & $20 \%$ \\
\hline 2 & 2.35 & $17.5 \%$ \\
\hline 4 & 4.76 & $19 \%$ \\
\hline 7 & 7.95 & $13.6 \%$ \\
\hline 9 & 10.0 & $11.1 \%$ \\
\hline 14 & 14.9 & $6.4 \%$ \\
\hline 20 & 19.9 & $0.5 \%$ \\
\hline 23 & 23.4 & $1.7 \%$ \\
\hline
\end{tabular}

After the stylus force of Alpha-Step 200 is calibrated, we then need to move the stylus to a right position so that a designed point along the free-standing structure will be scanned under the known stylus force. The designed point, in our case, is chosen to be at where maximum deflection of the freestanding structure happens. For example, centers are chosen for doubly-supported microbridges. Experimentally, the right scanning position of a microstructure can easily be found using positioning features fabricated by both sides of the microstructure. The stylus is at the right position only when it can scan over both positioning features (shown by the plot generated by the profiler). At the right position, a slow scan then can be done to measure the height $h$ of the microstructure under a known stylus force. In order to demonstrate the effects of structural loading, Fig. 2 shows schematically a profiler stylus that scans over a typical structure in which the cross section is symmetric about its center. As the profiler scan begins (Fig. 2a), a record is made proportional to the substrate-surface elevation. In Fig. $2 b$, the stylus is dragged up one side of the free-standing structure causing it to tilt to the left because of the bending moment by the off-axis loading. As the stylus proceeds to the central axis of the structure, the tilting decreases due to a smaller bending moment and the stylus height increases. The height reaches a maximum when the stylus is at the cross-sectional center (Fig. 2c) since the structure is loaded only by vertical force and not subjected to any twisting moment. After the stylus scans past the center, tilting in the opposite direction occurs which increases (decreasing $h$ ) until the stylus drops to the substrate (as shown in Fig. 2d). The height vs. position plot generated from the scan, therefore, has a maximum at the center of moment of the structure and a minimum at either edge. Thus, a profiler record indicates the central axis of a loaded member as the location at which $h$ is a maximum.

In order to measure the Young's modulus of the material under test, different stylus forces are used and the term $\frac{d h}{d F}$ is extracted from the data. In the following, two examples of the method are illustrated; Young's modulus is extracted for lowstress silicon nitride (using a doubly supported beam) and for polycrystalline silicon (using a bridge-slider structure).

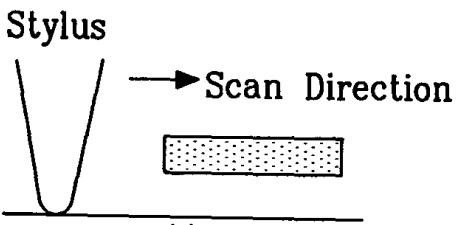

(a)

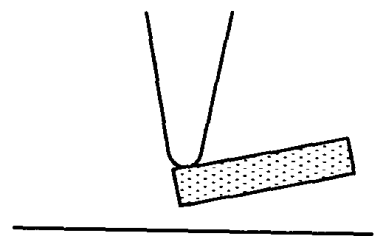

(b)

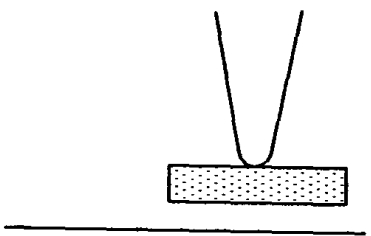

(c)

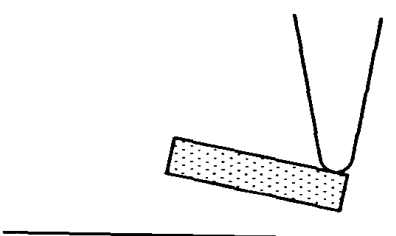

(d)

Figure 2 Schematic showing stages of a stylus scan over a free-standing structure. 

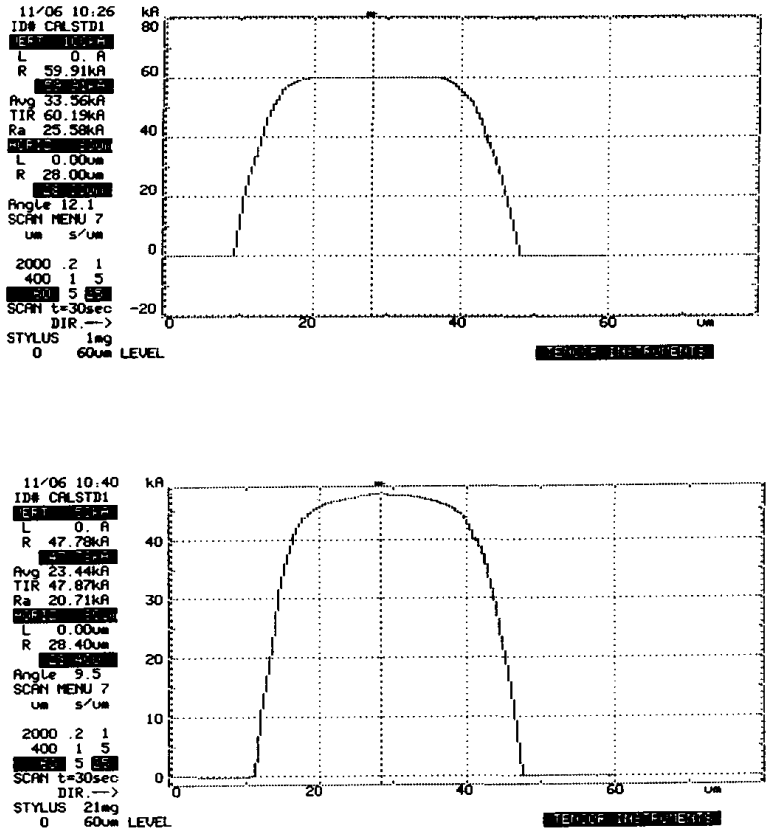

Figure 3 Typical height versus position plots generated from a surface profiler, Alpha Step 200.

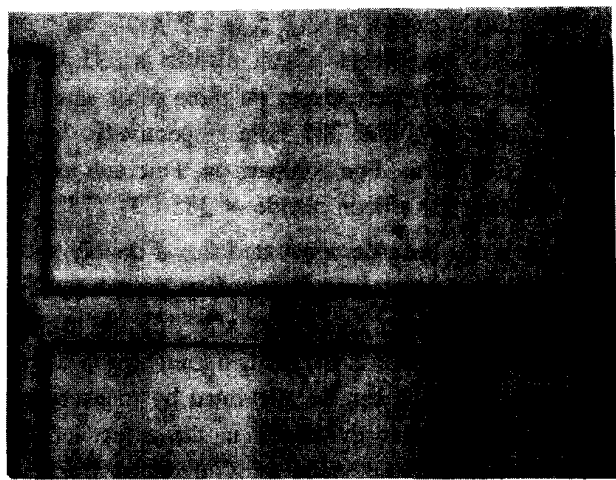

Figure 4 Top-view photograph of a doubly-supported, low-stress, silicon-nitride bridge fabricated with one mask. The bridge is $200 \mu \mathrm{m}$ long, $20 \mu \mathrm{m}$ wide, and $2.25 \mu \mathrm{m}$ thick. The undercut of the bridge supports (roughly half the bridge width) can be seen clearly because the silicon nitride is transparent. The shape of the undercut is used to establish the correct boundary conditions for simulations.

\section{LOW-STRESS SILICON NITRIDE}

Figure 3 shows typical height-versus-position plots generated by an Alpha Step 200 used on a low-stress microbridge shown in Fig. 4. The bridge is $200-\mu \mathrm{m}$ long, 20- $\mu \mathrm{m}$ wide and $2.25-\mu \mathrm{m}$ thick and the stylus force for the scan in Fig. 3a is set to its minimum value (about $1 \mathrm{mg}$ ). As seen in Fig. 3a, the profile scanned has a broad flat top which means that the $1 \mathrm{mg}$ force does not cause appreciable bending as it traverses the top of the bridge. In comparison, the tilting of the bridge can be clearly seen in Fig. 3b, which is a profile plot made with stylus force increased to roughly $21 \mathrm{mg}$. In general, Fig. 3 shows the symmetric height/position plots expected from our discussion in the previous section. The figure demonstrates that maximum heights can be easily extracted from the profiler traces.

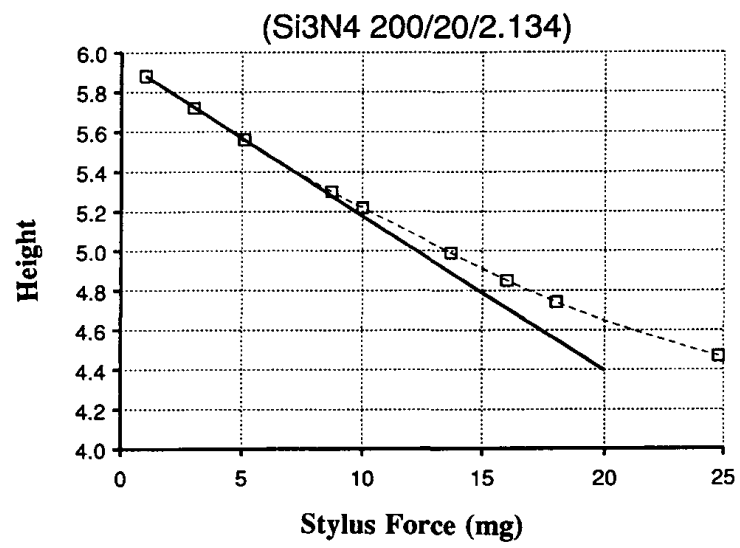

Figure 5 Height versus stylus-force plot of a low-stress, silicon-nitride bridge, $200-\mu \mathrm{m}$ long, $20-\mu \mathrm{m}$ wide, and 2.13- $\mu \mathrm{m}$ thick.

Plotted (as squares) in Fig. 5 are the results of measurements of $h$ using various loads on a silicon-nitride bridge measuring 200- $\mu \mathrm{m}$ long, $20-\mu \mathrm{m}$ wide, and $2.13-\mu \mathrm{m}$ thick. The stylus forces used range from 1 to $25 \mathrm{mg}$. The solid line in Fig. 5 is the tangent $\frac{d h}{d F}$ of the data curve at small stylus force. As the force increases, the tangent becomes smaller in magnitude because the bridge stretches. This behavior is general and it is therefore important to note that for an accurate measurement of Young's modulus using Eq. (2), the tangent must be obtained at very small loads. If $\frac{d h}{d F}$ is measured under large deflection, the Young's modulus will be significantly overestimated ( $\frac{d h}{d F}$ underestimated). As shown in Fig. 5, the slope $\frac{d h}{d F}$ is $-7.96432 \times 10^{-3} \mathrm{~m} / \mathrm{N}$ for small loads.

At this point, we must obtain the proportionality constant $K$ introduced in Eq. (2) in order to calculate $E_{Y}$. Before describing the extraction of $K$, we discuss in more detail the fabrication of our beam specimens. 
Only one mask is needed to make the silicon-nitride beams starting with wafers that are first coated with a $4-\mu \mathrm{m}$ thick phosphosilicate glass (PSG). After a $2-\mu \mathrm{m}$ thick layer of low-stress silicon nitride [11] is deposited, the mask is used to define the bridge pattern, and a timed etch of the PSG in buffered hydrofluoric acid (BHF) finishes the process. The wide supporting posts for the bridges are undercut by the BHF etch roughly one-half the bridge width. The undercutting of the supports is easily seen in Fig. 4 because silicon nitride is transparent. The visible undercut shows that these bridges are not exactly characterized by ideal clamped-clamped boundary conditions. Besides having nonideal boundary conditions, they also are subject to an invisible nonideality, tensile residual stress in the silicon nitride. In order to consider both the real boundary conditions and the effects of residual stress, a finiteelement simulator (SUPERSAP [8]) is used to calculate $K$. The input to SUPERSAP is the coordinates of the rectilinear top view (yz-plane) shown in Fig. 6a, which represents the beam shown in Fig. 4. It is also necessary to specify an initial estimate for $E_{Y}$.

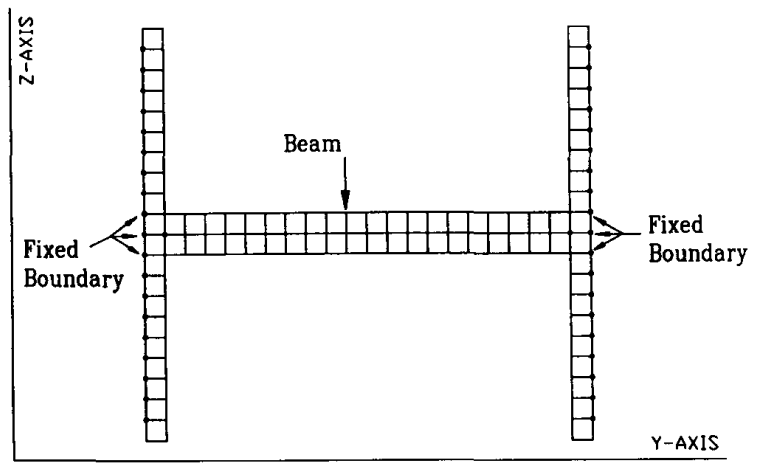

(a)

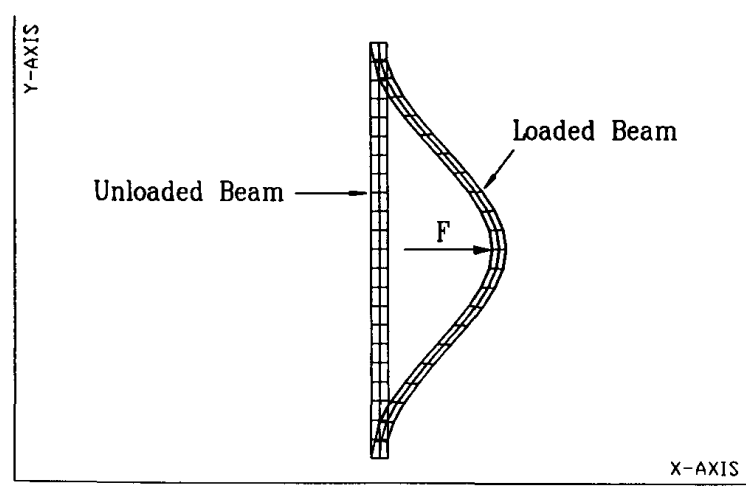

(b)

Figure 6 Finite-element structures for simulating $K$, (a) top view (yz-plane), (b) unloaded and pointloaded side views ( $x y$-plane).
Because there is no procedure to enter a value for residual stress directly into the initial conditions for SUPERSAP, its value in the beam is simulated through an artificial use of an elevated temperature. The stress has to be obtained by separate measurements. In our case this was done with a stylus-force measurement [1]. The stress is related to $E_{Y}$ by

$$
\sigma_{o}=(\alpha \Delta T) E_{Y}
$$

where $\sigma_{o}$ is the residual stress, $\Delta T$ represents a global temperature variation, and $\alpha$ is the thermal-expansion coefficient of the silicon nitride. To carry out the simulation, $(\alpha \Delta T)$ is varied to enter the value of the known residual stress. The residual stress used in the simulation is $100 \mathrm{MPa}$ [10] and the Poisson's ratio is assumed to be $0.3 . K$.

The iterating procedure is as follows. First, a guessed Young's modulus $E_{Y}$ is chosen for simulation and the residual stress $\sigma_{o}$ of $100 \mathrm{MPa}$ is entered by $(\alpha \Delta T)$ calculated using Eq. (3). A 1-mg point force is simulated as a load at the center of the bridge as shown in Fig. $6 \mathrm{~b}$ and the deflection at the loading point is calculated. Fig. $6 \mathrm{~b}$ shows both the unloaded and pointloaded side views of the bridge viewed from the side ( $x y$ plane). The constant $K$ is then calculated using Eq. (1). Then, using Eq. (2), $E_{Y}$ for a second iteration is calculated from this $K$ value and the experimental value of $\frac{d h}{d F}$ shown in Fig. 5 . Iterations continue until the starting $E_{Y}$ is the same as the iterated $E_{Y}$. For the beam shown in Fig, 4, only three iterations are needed to converge because measurements are available at very small loads and deflections. The value of $K$ simulated for the sample shown in Fig. 5 is $2.953 \times 10^{9} \mathrm{~m}^{-1}$ and the convergent $E_{Y}$ for low-stress silicon nitride is $371 \mathrm{GPa}$. We have carried out similar procedures on three other samples, and found $E_{Y}=365,367$, and $387 \mathrm{GPa}$ respectively; hence, the average $E_{Y}$ is $373 \mathrm{GPa}$. For comparison, Petersen as reported a Young's modulus for silicon nitride of $385 \mathrm{GPa}$ [13].

Although it has been demonstrated that a doubly supported bridge with residual stress can be used to determine $E_{Y}$, this structure suffers from two drawbacks affecting the accuracy of the procedure. First, there is the bridge-stretching problem discussed previously, which can be alleviated by measurements at very low loads. Another drawback is the effect of residual stress which influences the value of $K$ deduced from simulations. For example, if residual stress were changed from 100 to $300 \mathrm{MPa}$ for the sample in Fig. 5, the $K$ calculated from simulation would decrease by $4.8 \%$ and $E_{Y}$ would decrease by 18 GPa.

In the following, the use of another structure, a bridgeslider, is described that avoids the two problems stated above.

\section{POLYCRYSTALLINE SILICON}

Polysilicon has a considerably smaller Young's modulus than silicon nitride $[2,6,7]$ and the cross-axial stretchinginduced problems are therefore more severe during $\frac{d h}{d F}$ measurement with polysilicon than with silicon nitride. 
We have therefore introduced the bridge-slider [12] shown in Fig. 7. In this structure, one end of the bridge is anchored to the substrate through the bridge support, but the other end is connected to a slider that is free to move in a direction parallel to the surface. The cross section of the free-moving slider is shown in Fig. 8. The left part of Fig. 8 shows the slider before the etch of the sacrificial silicon-nitride layer. The right part is after the etch. Fig. 8 clearly shows that after the freeing etch, the slider is fully separated from the guiding plate by the thickness $(200 \mathrm{~nm})$ of the sacrificial silicon-nitride layer. The one-

dimensional, translational degree of freedom of the slider is thus achieved. Fabrication of the bridge-slider structure takes five masks and two layers of polysilicon. There are two advantages to this structure: (1) it releases any residual stress that may otherwise be present along the length of the beam, and (2) it prevents bridge stretching because one end is free.

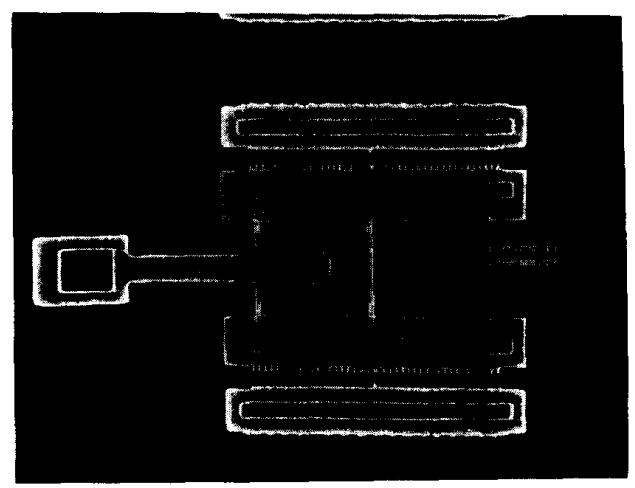

Figure 7 SEM photograph of a bridge-slider structure, $80-\mu \mathrm{m}$ long, $20-\mu \mathrm{m}$ wide, and $1.27-\mu \mathrm{m}$ thick. The marker is $80-\mu \mathrm{m}$ long.

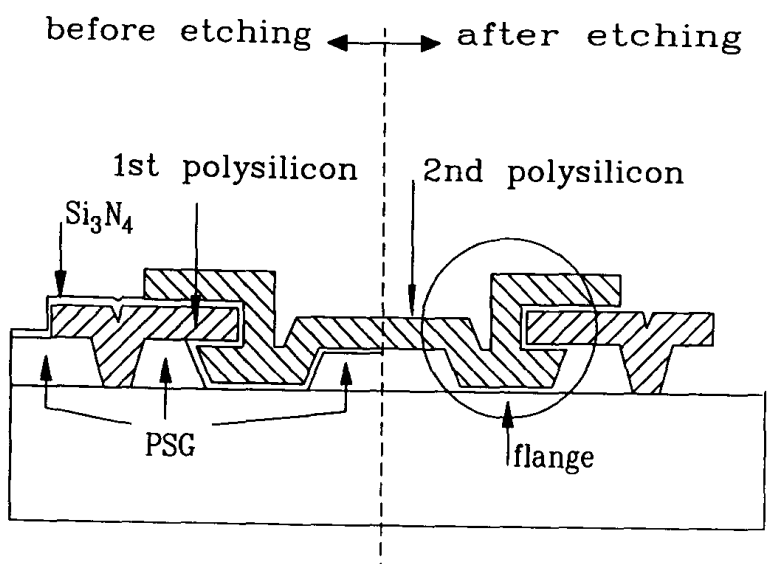

Figure 8 Cross section of the slider to demonstrate its one-dimensional, translational degree of freedom.
Figure 9 is a plot similar to Fig. 5 showing height versus load for a polysilicon bridge-slider that is $60-\mu \mathrm{m}$ long, $20-\mu \mathrm{m}$ wide, and $1.27-\mu \mathrm{m}$ thick. The polysilicon is heavily doped with phosphorus, and the highest temperature in its fabrication is $650^{\circ} \mathrm{C}$ (which happens during polysilicon deposition). The measured data in Fig. 9 all fall on the linear regressional line in contrast to the data in Fig. 5 showing that stretching of the beam is eliminated. The slope of the regressional line is $5.5611 \times 10^{-3} \mathrm{~m} / \mathrm{N}$. We can obtain $K$ for this bridge similarly to our procedures for the silicon-nitride bridge. However, it is easier in this case because residual stress need not be considered so only one simulation is needed. Considering only boundary conditions, $K$ is simulated to be $6.84 \times 10^{8} \mathrm{~m}^{-1}$ for the sample and $E_{Y}$ is $123 \mathrm{GPa}$ as calculated from Eq. (2). Measurements were then done on another bridge-slider with a beam 70- $\mu \mathrm{m}$ long, $20-\mu \mathrm{m}$ wide, and 1.27- $\mu \mathrm{m}$ thick and $E_{Y}$ for this sample was found to be $122 \mathrm{GPa}$, in excellent agreement with the first measurement.

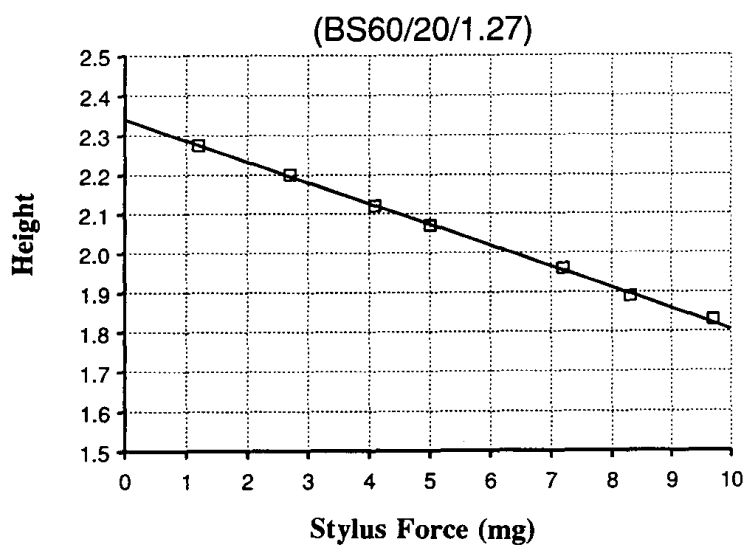

Figure 9 Height versus stylus-force plot of a polysilicon bridge-slider. The bridge slider is $60-\mu \mathrm{m}$ long, $20-\mu \mathrm{m}$ wide, and $1.27-\mu \mathrm{m}$ thick.

\section{CONCLUSION}

A straightforward and convenient method to measure the Young's moduli of thin-film materials is introduced. This method uses a surface profiler to measure the stylus force and structural deflection of a loaded free-standing structure. A finite-element simulator is used to consider nonideal boundary conditions and to account for any residual stress.

Experiments with doubly supported, low-stress, siliconnitride bridges are described first to demonstrate this method and $E_{Y}$ is found to be $373 \mathrm{GPa}$. Residual stress and excessive loading can complicate considerations of the data obtained on doubly supported beams. A bridge-slider structure is therefore introduced to avoid these influences and improve accuracy. From our experiments on a bridge-slider, the $E_{Y}$ of heavily phosphorus-doped polysilicon is found to be $123 \mathrm{GPa}$. The method described can also be applied to other microstructures such as cantilevers and diaphragms. 


\section{ACKNOWLEDGEMENTS}

We thank M. Lim and W. Tang for their help with SUPERSAP simulations. We also thank K. Voros, R. Hamilton, and the staff of the Berkeley Microfabrication Laboratory for assistance in processing.

\section{REFERENCES}

[1] A.S. Korhonen, P.L. Jones, and F.H. Cocks, "On the thermoelastic properties of hydrogenated amorphous silicon," Materials Science and Engineering, 49, 127(1981).

[2] S.P. Murarka and T.F. Retajczyk, Jr., "Effect of phosphorus doping on stress in silicon and polycrystalline silicon," J. Appl. Phys. 54 (4), 2069(1983).

[3] O. Tabata, K. Kawahata, S. Sugiyama, and I. Igarashi, "Mechanical property measurements of thin films using load-deflection of composite rectangular membrane," Proceedings of IEEE Micro Electro Mechanical Systems, pp. 152-156, Salt Lake City, Utah, Feb. 20-22, 1989.

[4] M.G. Allen, M. Mehregany, R.T. Howe, and S.D. Senturia, "Microfabricated structures for the in situ measurement of residual stress, Young's modulus, and ultimate strain of thin films," Appl. Phys. Lett. 51 (4), 241(1987)

[5] K.E. Petersen and C.R. Guarnieri, "Young's modulus measurements of thin films using micromechanics," $J$. Appl. Phys. 50 (11), 6761(1979).

[6] D.W. Deroo, "Determination of Young's modulus of polysilicon using resonant micromechanical beams," M.S. Thesis, Department of Electrical and computer engineering, University of Wisconsin-Madison, January 1988.

[7] M.W. Putty, S.C. Chang, R.T. Howe, A.L. Robinson, and K.D. Wise, "One-port active polysilicon resonant microstructures," Proceedings of IEEE Micro Electro Mechanical Systems, pp. 60-65, Salt Lake City, Utah, Feb. 20-22, 1989.

[8] SUPERSAP, Algor Interactive Systems, Inc., Essex House, Pittsburgh, Penn. 15206.

[9] Alpha Step 200, Tencor Co. Milpitas, CA.

[10] Y.C. Tai and R.S. Muller, "Integrated stylus-force gauges," Abstracts, Transducers' 89, the 5th Intl. Conf. on Solid-State Sensors and Actuators Montreux, Switzerland, pp. 158-159, June 25-30,1989.

[11] C.H. Mastrangelo, Y.C. Tai, and R.S. Muller, "Thermophysical properties of low-residual stress, siliconrich, LPCVD silicon nitride films," Sensors and Actuators, to be published.
[12] Y.C. Tai and R.S. Muller, "Fracture strain of LPCVD polysilicon," Tech. digest, IEEE Solid-State Sensor and Actuator Workshop, pp. 88-91, Hilton Head Island, South Carolina, June 6-9, 1988.

[13] K.E. Petersen, "Silicon as a mechanical material," Proceedings of the IEEE, Vol. 70, No. 5, 420-457, May, 1982. 